\title{
Avaliação do desempenho de corte de diferentes grãos adiamantados
}

\section{(Evaluation of the cutting performance of different types of diamond grains)}

\author{
E. C. Bianchi', P. R. de Aguiar ${ }^{1}$, E. J. da Silval, C. A. de Freitas ${ }^{2}$ A. R. R. Bianchi ${ }^{2}$ \\ ${ }^{1}$ Departamento de Engenharia Mecânica/Elétrica, Faculdade de Engenharia da UNESP, Campus de \\ Bauru, C. P. 473 Bauru, SP, 17033-360 \\ ${ }^{2}$ Departamento de Materiais Dentários, Faculdade de Odontologia de Bauru, \\ Universidade de S. Paulo, C. P. 73, Bauru, SP
}

\begin{abstract}
Resumo
Por meio de um trabalho multidisciplinar entre Engenheiros e Dentistas, foram estudados oito diferentes tipos de grãos abrasivos de diamantes para a fabricação de pontas adiamantadas e/ou rebolos de pequeno diâmetro. Verificou-se qual o grão mais adequado para ser empregado nas pontas, as quais são utilizadas em equipamentos que possuem um pequeno torque disponível. Uma metodologia de experimentação para avaliação da capacidade de corte destas ferramentas foi desenvolvida, utilizandose o vidro como material a ser desgastado. Como resultados, observou-se que a estrutura e a friabilidade do grão de diamante afetam diretamente a capacidade de corte das pontas adiamantadas. Desta forma, o melhor tipo de diamante foi aquele que apresentou, ao mesmo tempo, as melhores condições de remoção de material e o menor preço.
\end{abstract}

Palavras-chave: Diamantes, retificação, grãos superabrasivos.

\begin{abstract}
This paper presents a multi-area research involving Dentists and Engineers, in which eight different types of diamond abrasive grains, which are used in dental burs (small diameter grinding wheels) were tested. The most appropriated grain to be used in burs was evaluated. These burs are used in dental equipment characterized by low torque and low spindle rotation. A test methodology was developed in order to evaluate the cutting capacity of these burs, using glass as a machining material. The results show that it was possible to verify that the grain structure and friability were the properties that affect the cutting capacity. Consequently, the best diamond grain is the one that presented, simultaneously, the best material removal conditions and the lowest price.
\end{abstract}

Keywords: Diamond, grinding, abrasive grain.

\section{INTRODUÇÃO}

Pontas adiamantadas e/ou rebolos de pequeno diâmetro são ferramentas abrasivas normalmente utilizadas em equipamentos que possuem elevadas rotações, baixo torque e pouca rigidez, se comparados a rebolos com diâmetros maiores. O acionamento destes instrumentos pode ser feito por turbina movida a ar comprimido ou motor elétrico. $\mathrm{O}$ acionamento a ar comprimido, em geral, proporciona menor valor de torque disponível e maior rotação. Para acionamento com motor elétrico, o torque disponível é maior; porém a rotação é inferior. Em ambos os casos o tipo de grão abrasivo utilizado para a fabricação das pontas é fundamental, devido à capacidade de corte destes durante o processo produtivo.

Com a evolução dos materiais e dos processos de fabricação, os grãos de diamante das pontas começaram a ser fixados à ferramenta através do processo de eletrodeposição ou metalização elétrica com material de união à base de níquel, cromo, ou níquel-cromo. Neste processo a ferramenta (catodo), de material metálico (geralmente aço inoxidável), é imersa numa solução contendo íons metálicos, juntamente com um eletrodo (anodo) do material metálico que se deseja depositar (geralmente níquel). Usando-se uma membrana porosa, grãos de diamante são mantidos em contato com o que será a superfície ativa da ferramenta. Submetem-se então ambos (cátodo e ânodo) a uma diferença de potencial de modo que os íons metálicos se depositem na superfície da ferramenta (cátodo). A deposição contínua destes íons vagarosamente cria uma camada que, por sua vez, envolve os grãos de diamante fixando-os à ferramenta. Isto cria uma superfície abrasiva composta de uma camada de grãos de diamante envolvidos parcialmente por um "ligante" metálico [1]. Tanto o diamante natural como o sintético pode ser empregado na fabricação destas ferramentas. A escolha do tipo mais adequado deve aliar desempenho e custo.

O presente trabalho tem como objetivo avaliar o desempenho de grãos superabrasivos de diamante por meio 
da determinação da capacidade de corte dos mesmos. Estes são montados em pontas adiamantadas de aplicação odontológica, através do processo de eletrodeposição de níquel. Uma metodologia de experimentação foi desenvolvida para o estudo comparativo da capacidade de corte de oito pontas adiamantadas, contendo cada uma um diferente tipo de grão.

\section{Escolha dos tipos de grão de diamante}

A dificuldade em se avaliar o comportamento de um grão de diamante é um problema que sempre preocupou fabricantes e usuários de ferramentas adiamantadas [2]. Embora alguns atributos importantes, tais como o tamanho, resistência a fratura e a estabilidade térmica do grão, têm sido reconhecidos como regras básicas na seleção do grão para uma determinada aplicação. A seleção do grão de diamante mais adequado tornase complexa. A primeira grande complexidade reside na dificuldade em se identificar a condição exata de tensões sobre um grão individual durante uma determinada aplicação. A natureza do processo de corte é influenciada por uma infinidade de grãos, a qual resulta em uma distribuição randômica das forças de corte operantes na interface grão-peça [2].

$\mathrm{O}$ advento do diamante sintético possibilitou a obtenção de grãos de diamante, com diferentes propriedades mecânicas e físicas modificando-se apenas o processo de síntese. Atualmente, é possível a produção de diamantes sintéticos na escala que abrange desde grãos menores que $3 \mu \mathrm{m}$ a grãos maiores que $3 \mathrm{~mm}$, possibilitando ainda uma imensa variedade de formas, resistência e estabilidade térmica. Essa diversidade de características permite que o diamante sintético seja utilizado em uma larga variedade de aplicações abrasivas, como o polimento de cerâmicas ou o corte das pedras de granito de alta dureza [2].

A seguir são apresentadas algumas características dos diamantes utilizados no presente trabalho, bem como sugestões de aplicação e uso [3].

Com a finalidade de auxiliar os fabricantes de ferramentas adiamantadas, a empresa De Beers Industrial Diamond Division classificou os grãos conforme a Tabela I, onde são encontradas indicações comparativas entre os 12 tipos de

Tabela I - Algumas características dos diamantes da série PremaDia segundo o catálogo (adaptado) da empresa De Beers Industrial Diamond Division [4].

(Table I - Some characteristics of PremaDia diamonds [4])

\section{Série PremaDia de abrasivos de diamante de primeira linha para a industria mecânica}

Resistência ao impacto das partículas à temperatura ambiente

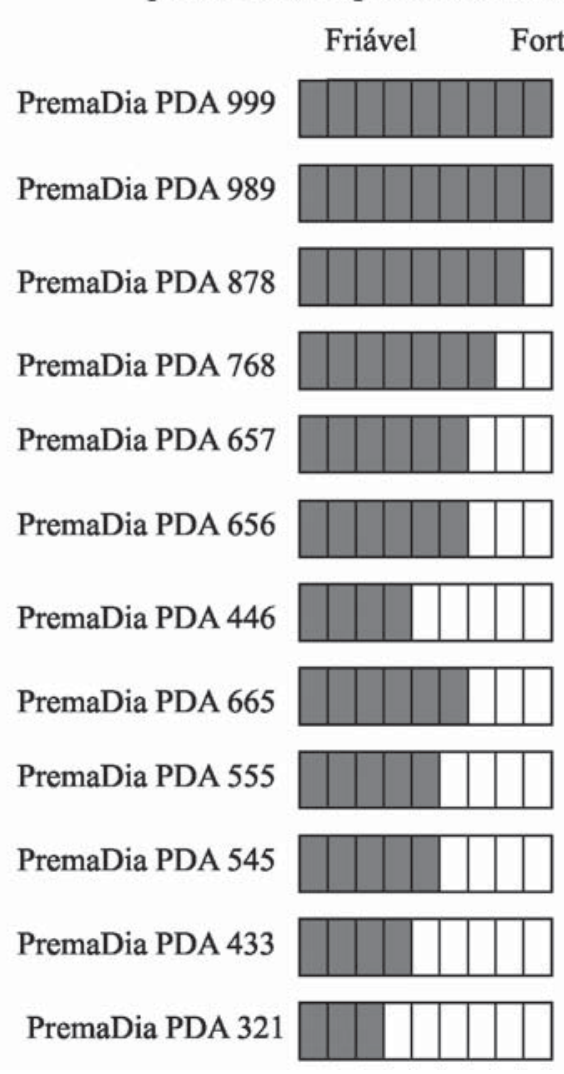

Resistência das partículas à temperatura elevada

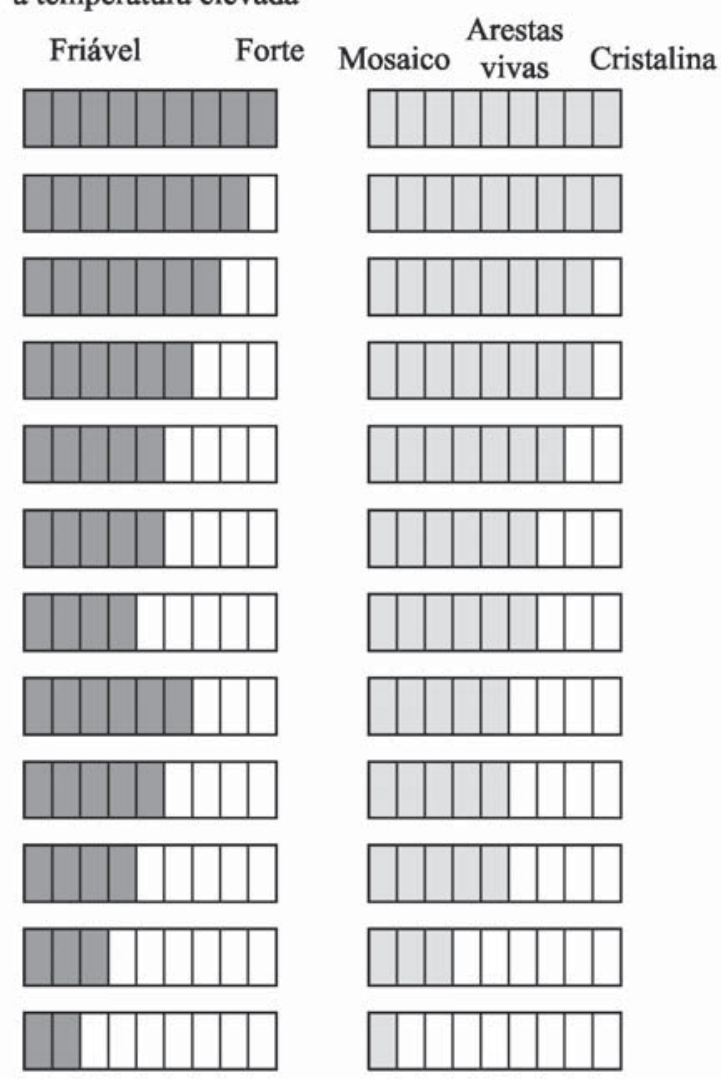


diamantes da série PremaDia (Diamantes de Primeira Linha). Para cada tipo, 3 diferentes propriedades são apresentadas, cada uma delas proporcionalmente quantificada dentro de uma série de 9 quadrículas, por meio de cores. Na primeira coluna é indicada a resistência do grão ao impacto à temperatura ambiente, na segunda, esta mesma resistência, após tratamento térmico controlado, e na última, à esquerda da tabela, a morfologia do grão, quanto à estrutura das partículas. Nesta última coluna, a estrutura cristalina é a mais pura (sem impurezas no cristalino, como um diamante para a confecção de jóias). Já a estrutura "mosaico" possui muitas impurezas e conseqüentemente é menos nobre e mais barato. O diamante PDA 321 é o que apresenta a menor classificação nas três grandezas relacionadas e o PDA 999 a maior classificação. O tipo mais adequado a uma determinada condição de trabalho será aquele que apresentar características de friabilidade (facilidade para fraturar o grão em pedaços, sob uma determinada força ou impacto) tais que possibilitem a existência permanente de arestas cortantes sobre a superfície da ferramenta e que não apresente desgaste demasiadamente acelerado.

Dentre os vários tipos de diamantes produzidos pela De Beers Industrial Diamond Division, listados na tabela I, foram escolhidos para serem avaliados apenas os identificados pelas siglas PDA 433, PDA 555, PDA 665, PDA 768 e PDA 878 visto que estes são representativos dos vários tipos básicos do conjunto.

Os diamantes da série PremaDia, segundo a empresa De Beers do Brasil Ltda., são apresentados, a seguir em ordem decrescente de friabilidade.

PDA 433: apesar de ser um produto eminentemente adequado para uso com ligantes resinóides, rotineiramente é fixado com níquel; constitui uma solução econômica para uma grande variedade de aplicações de uso geral. Trata-se de um diamante sintético, sendo o mais friável dentre todos os testados, segundo catálogo PremaDia NC1000793, da empresa De Beers Industrial Diamond Division.

PDA 555: projetado para aplicações em que se necessite de uma partícula (grão) mais friável, é um abrasivo de diamante natural, de partículas compactas e com formas irregulares; tem alta resistência ao choque térmico e as características do poder cortante dos diamantes naturais.

PDA 665: é afetado apenas a temperaturas extremamente elevadas, como todo o diamante natural; é considerado de primeira classe, por apresentar partículas compactas e bem formadas, presta-se a muitas aplicações de retificação, e suas características o fazem eminentemente adequado para a fabricação de ferramentas por eletrodeposição.

PDA 768: apresenta partículas de arestas vivas, selecionadas especialmente, assim como algumas partículas cristalinas compactas. A combinação particular de formas faz com que esse produto seja adequado para uma grande diversidade de aplicações, as quais não requerem um material de resistência muito elevada como o PDA 878.

PDA 878: é um abrasivo de uso geral, destinado a ser utilizado com ligante metálico. A estrutura da partícula é classificada como cristalina, com alta resistência ao impacto a temperaturas ambiente ou elevadas; foi concebido para apresentar um bom comportamento em uma grande variedades de aplicações, com ligante metálico; sua propriedades estão compreendidas entre as do PDA 768 e as do PDA 989.

Além dos diamantes da série PremaDia, foram testados os seguintes diamantes: TED-II: abrasivo com friabilidade e características apropriadas para fabricação de ferramentas por eletrodeposição, onde máquinas de torque razoavelmente elevado são utilizadas; DMB-G: aplica-se a ferramentas de torque não muito elevado, apresentando friabilidade pouco mais elevada que o diamante PDA-555; DMB-T: apresenta propriedades semelhantes ao diamante DMB-G, porém é pouco mais friável.

\section{MATERIAIS E MÉTODOS}

Para o estudo comparativo do desempenho de diferentes tipos de grãos de diamante empregados na fabricação de pontas adiamantadas, foram fabricados rebolos de pequeno diâmetro recobertos com grãos superabrasivos de diamantes naturais e sintéticos (granulometria D120/140). Utilizou-se o processo de eletrodeposição de níquel para a fixação dos grãos de diamante na parte ativa da haste metálica, cujo comprimento útil de utilização é de $12 \mathrm{~mm}$. O controle da distribuição dos grãos de diamante ao longo deste comprimento útil depende das variáveis do processo de fabricação e das características das partículas, tais como: tamanho médio dos grãos, intensidade de corrente galvânica, dentre outros. Estas pontas adiamantadas foram ensaiadas em um banco de ensaio composto por três partes básicas: mecânica, eletrônica e computacional.

A parte mecânica do banco de ensaios é constituída de uma máquina retificadora plana Reti Lite, com $600 \mathrm{~mm}$ de curso longitudinal, $400 \mathrm{~mm}$ de curso transversal e com 400 $\mathrm{mm}$ de altura entre o rebolo e a mesa. O movimento transversal da mesa é controlado por um motor de corrente contínua com velocidade mínima de $20 \mathrm{~mm} / \mathrm{min}$ e máxima de $500 \mathrm{~mm} / \mathrm{min}$. O movimento longitudinal da mesa é feito por meio de um sistema hidráulico.

No cabeçote da retificadora foi fixado um micromotor Dentec de corrente contínua de $31 \mathrm{~V}$ de tensão de alimentação apresentando rotação máxima de $24.000 \mathrm{rpm}$. A fixação da peça ao cabeçote foi feita com o auxílio de uma peça de nylon, elaborada para que a caneta não se danifique durante o processo de usinagem. Sobre a mesa da máquina retificadora, utilizouse uma morsa para a fixação dos corpos-de-prova.

A parte eletrônica do banco de ensaios é responsável pela aquisição dos valores de tensão e corrente elétrica que agem sobre o micromotor, e conversão desses valores em intensidades compatíveis com a placa de aquisição A/D Novus, com tempo de conversão de $17 \mathrm{~ms}$.

A conversão de valores pela parte eletrônica da montagem consistiu na conversão do valor de corrente consumida pelo micromotor em tensão compatível com a placa de aquisição $\mathrm{A} / \mathrm{D}$, conversão do valor de tensão aplicada sobre o micromotor em valores compatíveis com a placa de aquisição A/D e conversão da freqüência de rotação do eixo do micromotor em tensão compatível com a placa de aquisição A/D.

$\mathrm{Na}$ placa de aquisição os valores de tensão foram 
transformados em sinais digitais, possíveis de serem interpretados pelo computador e processados por um software desenvolvido para a realização desse trabalho.

O objetivo do software é tratar matematicamente e armazenar em arquivos os valores enviados pela placa de aquisição, a fim de se obter em tempo real a intensidade da força tangencial de corte. Ao final da realização de cada cálculo, o software está habilitado a identificar um ponto, que representa a intensidade da força de corte, em um gráfico da força tangencial de corte em função do número de passos, apresentado diretamente no monitor de vídeo do computador.

$\mathrm{O}$ valor da força tangencial de corte $\left(\mathrm{F}_{\mathrm{t}}\right)$ da ferramenta pode ser obtido pela equação:

$$
\mathrm{T}=\mathrm{F}_{\mathrm{t}} \times\left(\mathrm{d}_{\mathrm{s}} / 2\right)
$$

onde $\mathrm{d}_{\mathrm{s}}$ é o diâmetro externo da ponta e $\mathrm{T}$ é o torque aplicado pelo micromotor que é obtido pela equação:

$$
\mathrm{T}=(\mathrm{I} \times \mathrm{V} \times 60) /(2 \times \pi \times \mathrm{n})
$$

sendo I e V os valores de corrente e tensão elétrica aplicados no micromotor da caneta odontológica, e n o número de rotações no eixo do micromotor. Logo, dois dos valores para a determinação da intensidade do torque instantâneo (T) são variáveis diretamente conhecidas, pois os valores de corrente elétrica instantânea (I) e tensão elétrica instantânea (V) são transformados pela placa A/D em sinais digitais e enviados ao software.

O número $n$ de rotações na unidade de tempo no eixo não é obtido diretamente pela montagem eletrônica, devido às dificuldades físicas para a instalação de um medidor de rotação como, por exemplo, um "encoder" no eixo árvore da caneta. Com a utilização dos valores de tensão e corrente elétrica instantâneos é possível determinar a rotação instantânea do eixo árvore do micromotor com a utilização de um modelo matemático, desenvolvido por [5], que simula o funcionamento do micromotor de corrente contínua da caneta odontológica.

Escolheu-se o vidro (temperado plano, tipo cristal) como material a ser usinado, pois esse apresenta características mais uniforme, fazendo com que se preste para o estudo da eficiência de corte de grãos de diamante [6]. Por gentileza da empresa Saint-Gobain Abrasivos. (S. Paulo, SP), tal material foi caracterizado como apresentando dureza média SPB de 3,98, após jateamento com quartzo de granulação 20/30 e pressão de $19,1 \mathrm{psi}\left(1,34 \mathrm{kgf} / \mathrm{cm}^{2}\right)$, por $30 \mathrm{~s}$, em uma área cilíndrica delimitada de $0,785 \mathrm{~cm}^{2}$. As dimensões dos corpos-de-prova são $155 \mathrm{~mm}$ x $90 \mathrm{~mm}$ x $8 \mathrm{~mm}$. O corte foi realizado no comprimento de corte foi de $90 \mathrm{~mm}$.

s pontas adiamantadas foram submetidas a ensaios de retificação do tipo tangencial plana de mergulho. Nesses ensaios a velocidade de deslocamento transversal da mesa da máquina retificadora (que coincide corpo a velocidade do corpo-de-provas) foi $0,0055 \mathrm{~m} / \mathrm{s}$, a velocidade de corte da ponta adiamantada $7,1 \mathrm{~m} / \mathrm{s}$ e a penetração da ponta adiamantada no corpo-de-provas foi de $400 \mu \mathrm{m}$ por passo. Estes valores foram mantidos constantes para a realização de todos os ensaios experimentais.

As pontas adiamantadas foram solicitadas até o momento em que, por elevação excessiva do torque, ocorria o travamento do eixo do micromotor (fim de ensaio). Conseqüentemente, como o comportamento de cada ponta adiamantada foi distinto, o volume de material removido (determinado pelo produto entre o número de passadas da ponta sobre o corpo-de-prova e a área superficial deste) diferenciou para cada ensaio realizado.

\section{RESULTADOS E DISCUSSÃO}

Pôde-se notar em todos os ensaios uma grande dispersão dos resultados num mesmo lote de pontas adiamantadas. Esse fato já era esperado, pois se sabe que as melhores pontas adiamantadas são aquelas onde há espaçamentos maiores e homogêneos entre os grãos de diamantes. As dificuldades em se assegurar a homogeneidade no processo de fabricação, por se tratar de uma eletrodeposição, não confere as mesmas qualidades para todas as pontas adiamantadas, mesmo que essas pertençam a um mesmo lote de fabricação. Sempre ocorrem diferenças relacionadas ao espaçamento médio entre os grãos, a densidade de grãos abrasivos de diamante, qualidade de Ni depositado, dentre outros.

Assim, em pontas com maiores espaçamentos o número de grãos ativos é menor e, conseqüentemente, os cavacos arrancados apresentam maiores espessuras. Dessa forma, os grãos abrasivos são submetidos a maiores esforços de corte, tendendo a desgastarem-se mais rapidamente e desprenderemse antecipadamente da superfície de corte da ponta. Ressaltase, todavia, que espaçamentos excessivos são prejudiciais a eficiência de corte da ponta.

Verificou-se que a tendência de remoção de material conferida às pontas confeccionadas com o diamante PDA-878 foi a menor entre todas. Dentre os diamantes estudados, este tipo de diamante é o que apresenta morfologia cristalina, com partículas de formato uniforme. Portanto, é o menos friável [4] (Ver Tabela I) dentre as pontas adiamantadas testadas. Assim, necessita de grandes esforços de corte para a geração de novas arestas no topo dos grãos abrasivos.

Considerando-se o pequeno torque disponível no eixo do micromotor utilizado neste trabalho, os valores de força tangencial de corte foram insuficientes para fraturar os grãos abrasivos. Desta forma, ocorreu elevação da área de contato entre os grãos abrasivos e o material, elevando rapidamente os valores de força tangencial de corte, o que causou o travamento do eixo do micromotor.

Em seqüência, as pontas confeccionadas com os diamantes TED-II, PDA-768, PDA-665, PDA-555, DMB-G, DMB-T e PDA-433, apresentaram progressivamente maior capacidade de remoção de material, devido as estruturas dos grãos apresentarem cada vez mais defeitos; ou seja, são respectivamente mais friáveis. Desta forma, há uma maior geração de novas arestas de corte no topo dos grãos abrasivos, fazendo com que as pontas permaneçam agressivas (com capacidade da ponta em remover material) por mais tempo. Na Tabela II são apresentados os valores de volume de material 
Tabela II - Valores de volume de material removido em todos os ensaios e análise estatística.

(Table II - Values of the removed material and statistical analysis)

\begin{tabular}{|c|c|c|c|c|c|c|}
\hline & \multicolumn{5}{|c|}{ Material Removido $\left(\mathrm{mm}^{3}\right)$ - valores em ordem crescente } & \\
\hline DMB-T & 1488 & 3472 & 16864 & 19344 & 19344 & \\
\hline PDA-878 & $\underline{1984}$ & 1984 & $\underline{2480}$ & 5952 & 5952 & \\
\hline PDA-555 & 992 & 992 & $\underline{2976}$ & 17460 & 23808 & 31744 \\
\hline PDA-433 & 2976 & $\underline{26784}$ & 40176 & 44144 & & \\
\hline PDA-665 & 998 & 1984 & 1984 & $\underline{8928}$ & 10416 & 34224 \\
\hline PDA-768 & 992 & $\underline{992}$ & 1488 & $\underline{3472}$ & 16368 & 28768 \\
\hline DMB-G & 6448 & 13392 & 13392 & 18352 & 21824 & \\
\hline \multirow[t]{7}{*}{ TED-II } & $\underline{1488}$ & $\underline{1984}$ & $\underline{3472}$ & 16368 & & \\
\hline & & & & & & \\
\hline & \multicolumn{6}{|c|}{$\begin{array}{l}\text { Em destaque os valores que se enquadram dentro dos limites de confiança, } \\
\text { são os mesmos valores utilizados para o cálculo da média }(\mu)\end{array}$} \\
\hline & & & & & & \\
\hline & & & & & & \\
\hline & & & & \multicolumn{2}{|c|}{ Limites } & \\
\hline & Média(x) & Variância & Erro & Inferior & Superior & Média $(\mu)$ \\
\hline DMB-T & 12102,4 & 8869,9 & 7774,8 & $\begin{array}{r}4327,6 \\
\end{array}$ & 19877,2 & 18517 \\
\hline PDA-878 & 3670,4 & 2092,6 & 1834,3 & 1836,1 & 5504,7 & 2149 \\
\hline PDA-555 & 12995,3 & 13243,2 & 10596,7 & 2398,6 & 23592 & 6812 \\
\hline PDA-433 & 28520 & 18578,5 & 18206,9 & 10313,1 & 46726,9 & 37034 \\
\hline PDA-665 & 9755,7 & 12627,9 & 10104,4 & 0 & 19860,1 & 4862 \\
\hline PDA-768 & 8680 & 11486,4 & 9191,1 & 0 & 17871,1 & 4662 \\
\hline DMB-G & 14681,6 & 5822,5 & 5103,6 & 9578 & 19785,2 & 15045 \\
\hline \multirow[t]{4}{*}{ TED-II } & 5828 & 7077,1 & 6935,3 & 0 & 12763,3 & 2315 \\
\hline & \multicolumn{3}{|c|}{ Média $(x)$ = média de toda a população } & & & \\
\hline & \multicolumn{6}{|c|}{ Média $(\mu)=$ média dos valores que se enquadram dentro dos limites de confiança } \\
\hline & \multicolumn{3}{|c|}{ Erro calculado para $95 \%$ de confiança } & & & \\
\hline
\end{tabular}

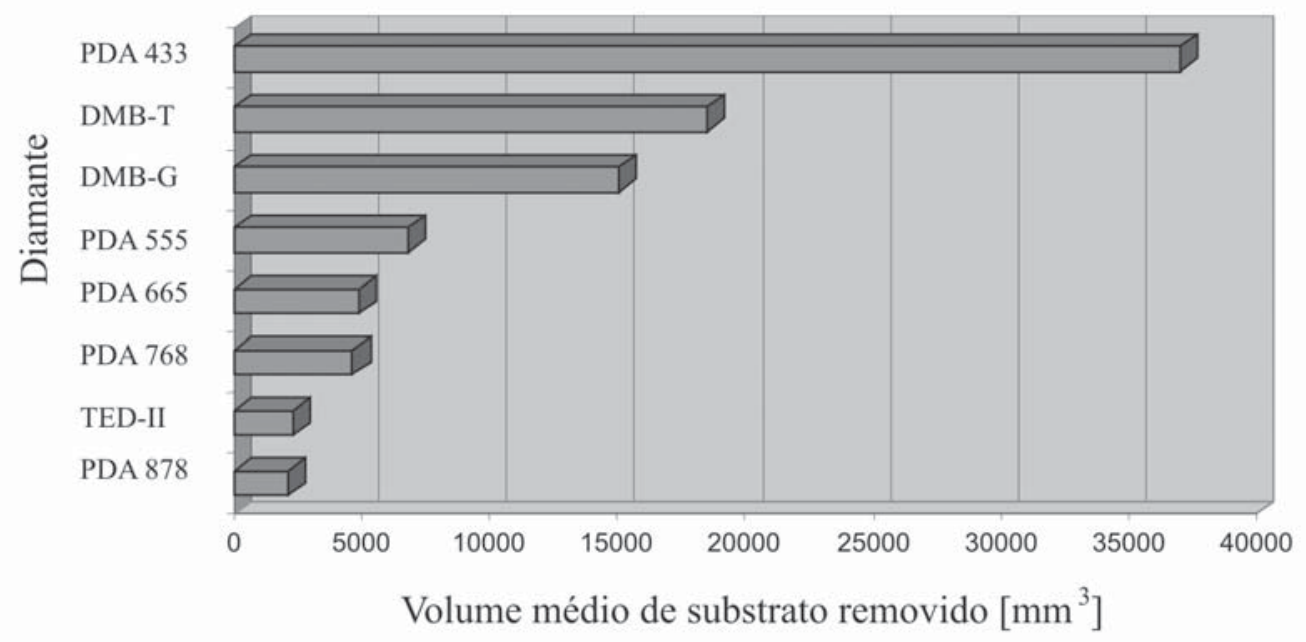

Figura 1: Volume médio de material removido em função do tipo de diamante utilizado. Figure 1: Average volume of the removed material for different types of diamonds.) 
removido em todos os ensaios de passagem realizados, com os quais foi desenvolvida uma análise estatística para $95 \%$ de confiança.

Posteriormente, foi elaborada a Fig. 1, relacionando o tipo de diamante utilizado com o volume médio de material removido, média $(\mu)$. Para o cálculo desta média, utilizaramse somente os valores que se enquadraram dentro dos limites de confiança inferior e superior (Tabela II).

Nota-se claramente que as pontas confeccionadas com o diamante PDA-433 apresentaram maior vida útil pelo melhor aproveitamento dos grãos. Isso somente foi possível pela maior friabilidade deste grão quando comparado aos demais testados (ver Tabela 1), fazendo com que a força tangencial de corte permanecesse num patamar inferior por mais tempo, com relação aos outros diamantes utilizados, removendo mais material antes que o valor limite de torque suportado pelo eixo do micromotor da caneta odontológica fosse atingido.

Pode-se verificar que as pontas confeccionadas com o diamante PDA-433 apresentaram uma capacidade significativamente superior na remoção de material, em relação aos outros diamantes. Ressalta-se que essa capacidade foi substancialmente maior se comparada a dos diamantes naturais PDA-555 e PDA-665, os quais são utilizados atualmente pela maioria das empresas na confecção de pontas odontológicas.

\section{CONCLUSÕES}

Utilizando-se equipamentos de baixo torque, a estrutura da partícula do diamante e, conseqüentemente, de sua friabilidade exercem grande influência no comportamento das pontas adiamantadas. Com a progressiva diminuição de defeitos na estrutura da partícula, o grão torna-se menos friável; assim, as forças de corte desenvolvidas não são suficientes para a geração de novas arestas no topo dos grãos abrasivos. Desta forma, há a elevação da área no topo dos grãos abrasivos, elevando a força tangencial de corte, o que limita a vida útil da ponta odontológica, pelo travamento do eixo do micromotor.

O diamante PDA-433 apresentou maior capacidade de remoção de material, em relação aos outros tipos de diamantes devido a sua maior friabilidade, a qual está associada à sua menor resistência ao impacto, resultado de sua menor dureza e pureza. A friabiliade do grão aliada ao menor custo em relação aos demais diamantes testados torna-o mais adequado para a fabricação de pontas utilizadas em instrumentos que possuem baixo torque.

\section{REFERÊNCIAS}

[1] A. K. Chattopadhyay, H. E. Hintermann, On Improved Bonding of TiC-Coated CBN Grits in Nickel-Based Matrix, Ann. of the CIRP 42, 1 (1993) 413-415.

[2] P. R. Davis, M. L. Fish, , S. Peacock, , D. N. Wright, An indicator system for saw grit, Industrial Diamond Review. 56, 570 (1996) 78-87,.

[3] M. W. Bailey, H. O. Juchem, Selection and use of PremaDia, Industrial Diamond Review (1994) 8-11

[4] De Beers Industrial Diamond Div., Cat. PremaDia. NC1000793, Berkshire, Inglaterra.

[5] P. R. Aguiar, I. Rotta, A simulação da máquina de corrente contínua a partir da determinação dos parâmetros - método não convencional, IV Congresso Latino Americano de Controle Automático, Puebla, México (1990) 946-950.

[6] J. C. Vaz, L. Steagall, M. Issao, Eficiência de instrumentos de diamante no desgaste do esmalte de dentes humanos e vidro, Ensaio preliminar com instrumentos nacionais e estrangeiros, Rev. Ass. Paul. Cirurg. Dent. 35, 1 (1981) 82-91. (Rec. 30/08/02, Rev. 23/06/03 e 17/10/03, Ac. 28/12/03) 